\title{
Spatial variation in maximum dive depth in gray seals in relation to foraging
}

Theoni Photopoulou, ${ }^{1,2}$ Sea Mammal Research Unit, Scottish Oceans Institute, University of St Andrews, Scotland KY16 8LB, United Kingdom and Centre for Research into Ecological and Environmental Modelling, The Observatory, University of St Andrews, Scotland KY16 9LZ, United Kingdom; Michael A. FedaK, Sea Mammal Research Unit, Scottish Oceans Institute, University of St Andrews, Scotland KY16 8LB, United Kingdom; LEN Thomas, Centre for Research into Ecological and Environmental Modelling, The Observatory, University of St Andrews, Scotland KY16 9LZ, United Kingdom; JASON MatThiopoulos, Institute of Biodiversity, Animal Health and Comparative Medicine, Graham Kerr Building, University of Glasgow, Glasgow, Scotland G12 8QQ, United Kingdom.

\begin{abstract}
Habitat preference maps are a way of representing animals' space use in two dimensions. For marine animals, the third dimension is an important aspect of spatial ecology. We used dive data from seven gray seals Halichoerus grypus (a primarily benthic forager) collected with GPS phone tags (Sea Mammal Research Unit) to investigate the distribution of the maximum depth visited in each dive. We modeled maximum dive depth as a function of spatiotemporal covariates using a generalized additive mixed model (GAMM) with individual as a random effect. Bathymetry, horizontal displacement, latitude and longitude, Julian day, sediment type, and light conditions accounted for $37 \%$ of the variability in the data. Persistent patterns of autocorrelation in the raw data suggest that individual intrinsic rhythm might be an important factor, not captured by external covariates. The strength of using this statistical method to generate spatial predictions of the distribution of maximum dive depth is its applicability to other plunge and pursuit divers. Despite being predictions of a point estimate, these maps provide some insight into the third dimension of habitat use in marine animals. The capacity to predict this aspect of vertical habitat use may help avoid conflict between animal habitat and coastal or offshore developments.
\end{abstract}

Key words: maximum dive depth, spatial variation, Generalized Additive Mixed Model, Halichoerus grypus, space use, habitat preference.

Knowing where and when marine predators dive to different depths is essential, because it is a measure of space use in the vertical dimension. The depth of dives is expected to vary with the type of prey that the animals encounter and exploit,

${ }^{1}$ Corresponding author (e-mail: theoni.photopoulou@uct.ac.za).

${ }^{2}$ Current address: Department of Statistical Sciences, University of Cape Town, Rondebosch 7701, Cape Town, South Africa and Animal DemographyUnit, University of Cape Town, Rondebosch 7701, South Africa. 
within the geographical and environmental space they can access. Depth also indicates where, in the water column, human and animal activities are likely to overlap. In the United Kingdom, it is currently a legal requirement to investigate the potential effect of coastal and offshore industry on the marine environment, and marine animals that are protected, such as seabirds and marine mammals. This is often done by generating spatial predictions of habitat use based on telemetry data, but these seldom present depth use. Two-dimensional habitat preference maps, defined here as spatially indexed predictions of occurrence, fail to characterize animals' three-dimensional habitat preferences and their chances of encountering anthropogenic activities at depth, such as marine renewable energy installations, at the construction or operational stages.

Due to technological constraints, dives by air-breathing animals are most often recorded and stored in discrete depth and time, and studied as units of foraging behavior. Successful foraging may be interspersed with unsuccessful foraging, or associated with other activities. The maximum depth visited during a dive is a statistic commonly returned by telemetry devices and used to describe diving behavior. Though it is a point estimate of the distribution of depth visited during a dive, this can be useful for species that feed benthically, such as gray seals (Halichoerus grypus) (Thompson et al. 1991) and European shags (Phalacrocorax aristotelis) (Wanless et al. 1993, Gremillet et al. 1998, Watanuki et al. 2008), where maximum dive depth could be considered a proxy for foraging, or attempted foraging, since they are known to feed at, or near the seabed. The motivation for using maximum dive depth, and not a derived metric of diving or foraging behavior, such as dive shape (e.g., Schreer and Testa 1996) or Area-Restricted-Search (ARS) (Fauchald and Tveraa 2003) was to determine what factors influence maximum dive depth itself. We explore the potential for using this simple, often readily available, metric to characterize diving behavior, and its relationship with environmental and individual covariates. Although the maximum depth visited in each dive does not describe the distribution of depths visited, knowing what proportion of variability is explained by maximum depth alone, might help with the development of methods that do.

Gray seals are generalist and opportunistic predators (Hammond et al. 1994a), so some foraging is likely to occur throughout time spent at sea, including during transit phases, between food patches and between haul-out sites. Previous tagging studies have shown substantial individual variation in areas visited, with animals passing through areas that other animals remain in, presumably to forage (McConnell et al. 1999). Dietary studies based on scat analysis have shown that gray seals forage mainly on sand eels or sand lance (Ammodytes spp.), gadids (Gadus, Melanogrammus, Merlangius, and Pollachius spp.), flatfish and sculpins (Solea and Platichthys spp., and members of the Cottidae family) as well as squid (Illex spp.) (Prime and Hammond 1990; Bowen and Harrison 1994; Hammond et al. 1994a, b). This suggests that gray seals feed on benthic and demersal prey throughout the year, though there is evidence for seasonal and geographic variation in diet composition (Prime and Hammond 1990; Bowen et al. 1993; Bowen and Harrison 1994; Hammond et al. 1994a, b; Beck et al. 2007).

In air-breathing divers, time spent at depth is limited by having to return to the surface to breathe even though various physiological techniques are used to relax this limitation by careful management of oxygen stores and metabolic adjustments (Kooyman and Ponganis 1998, Kanatous et al. 1999, Sparling and Fedak 2004, Meir et al. 2009). Gray seals often forage at, or near the seafloor (Thompson et al. 1991) and, because diving involves expenditure of time and energy despite diving adaptations, increased distance to and from the bottom leads to increased energy 
expenditure and reduced potential time at depth. As a consequence, we expect to see a positive relationship between maximum dive depth and the quality of foraging habitat in gray seals. Two complications to testing this are the hard limit to dive depth presented by the seafloor and the relatively shallow depth of the North Sea. Investigating the spatial variability in maximum dive depth is useful because it might help to identify the elements that determine the quality of foraging habitat and define important foraging areas at sea (e.g., Aarts et al. 2008) and can help quantify the encounter rate between animals and coastal or offshore developments. In the absence of detailed information on prey distribution, we investigate how the maximum depth of dives of gray seals during trips to sea is affected by individual, spatial, temporal, and physical environmental variables using a generalized additive mixed model (GAMM).

\section{Material And Methods}

\section{Exploratory Data Analysis and Data Processing}

The data set was collected using GPS phone tags (SMRU), deployed on seven gray seals at Abertay Sands $\left(56^{\circ} 26.17^{\prime} \mathrm{N}, 2^{\circ} 47.10^{\prime} \mathrm{W}\right)$ in April 2008, and consisted of approximately 335,000 dives in total (Table 1). The tags were glued to the hair of the seals, behind the head, using fast-setting epoxy resin (Fedak et al. 1983, McConnell et al. 1999). The tag software defined behavioral states as follows: state "hauled out" starts if the tag is dry for $10 \mathrm{~min}$, and ends if the tag is wet for at least $40 \mathrm{~s}$; state "diving" starts if the tag is wet and depth is greater than $1.5 \mathrm{~m}$ for $8 \mathrm{~s}$, and ends if depth is less than $1.5 \mathrm{~m}$ for any length of time $(0 \mathrm{~s})$, or dry at any time. In this program "at the surface" is the complement of "hauled out" and "diving." When in state "diving," depths are collected every $4 \mathrm{~s}$. Once the dive-end criteria are met, the depth data are reduced to 11 depth points: one at the beginning and end of the dive, and nine at equal time intervals throughout the dive. The maximum depth reached during the dive is also collected and stored. The series of maximum dive depths, one for each dive of each individual, were analyzed.

Telemetry data show that although seals spend a large proportion of their time relatively close to a haul-out site, they can also engage in long-distance travel (McConnell et al. 1999; SMRU, unpublished data): this was also observed in this study. While at sea, seals travel to offshore areas and also move between haul-out sites. When observed during real time tracking, (Thompson et al. 1991) these were assumed to be foraging trips, because seals were seen in association with other marine predators. During these trips, seals swam slowly and dived to near the seabed.

To isolate the dive data that were directly relevant to foraging behavior, track data were classified into segments of "trip," "transit," and "haul-out." To do this, the track and dive record of each individual was visually inspected in MamVIS AD (Fedak et al. 1996) and Google Earth (Google Inc. 2009), and segments of the time series that corresponded to trips to sea were selected for analysis. A trip started at the first at-sea location of a direct trajectory away from a haul-out site, and ended at the first on-land location of a haul-out period. A haul-out period started at the first dry, onland location of a dry period lasting at least three hours to make trips clearly distinguishable, and ended at the onset of a trip. A transit was a trip that started and ended at different haul-out sites and showed directed movement between the two without excursions into offshore areas away from that trajectory. There were few transits 


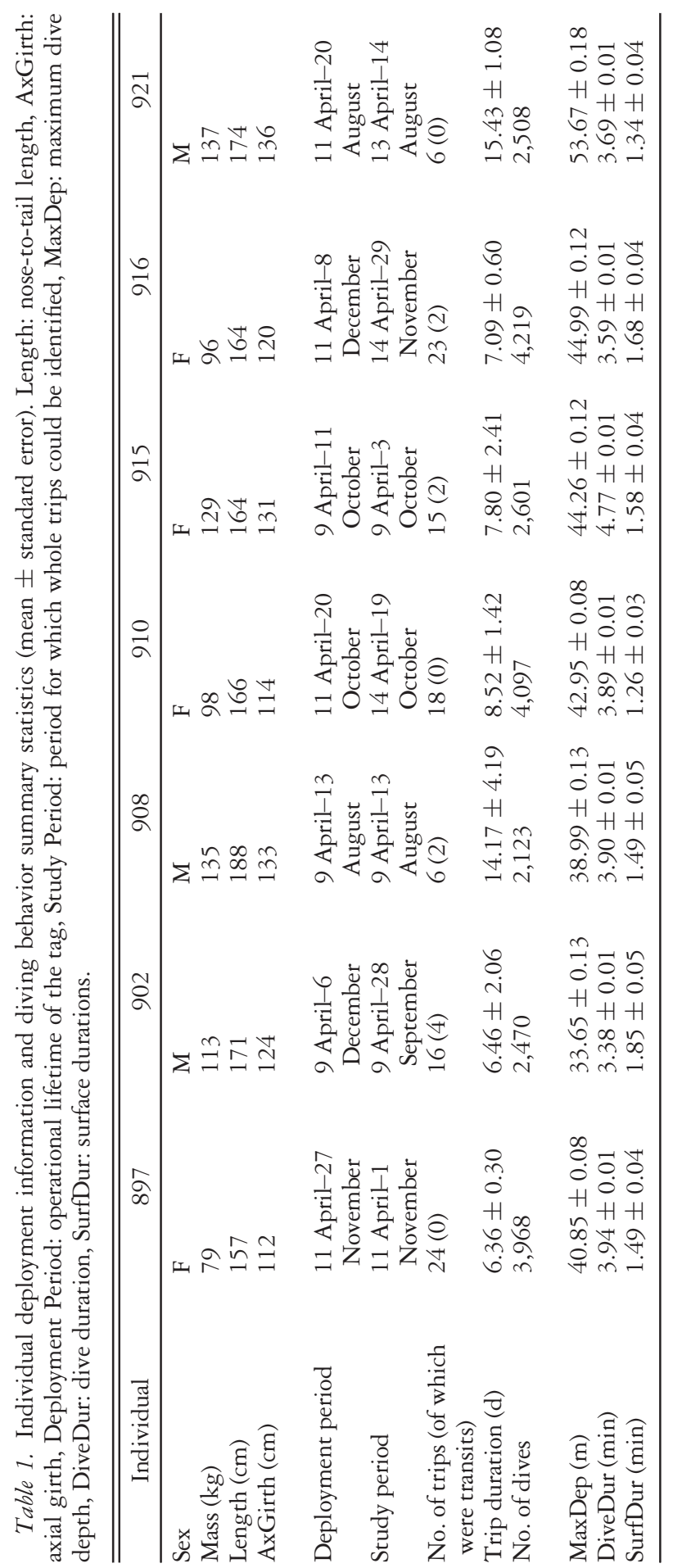


identified in this data set and they were included in the maximum dive depth analysis as trips since foraging cannot be ruled out during this type of travel. Tracks were classified manually according to these criteria for individual tracks and associated dive records.

The number of trips identified per individual ranged from 6 to 24 (Table 1). Exploratory analysis of the trip data showed that animals that made more trips had a bigger proportion of shallow maximum dive depths than animals that made few trips. This is because, assuming dives always reached the bottom in shallow areas, animals that made more trips, and whose trips were shorter in duration, came within close range of the shore more often, and as a result encountered depths of $<20 \mathrm{~m}$ more frequently. The data set of trips and transits consisted of over 200,000 dives.

Telemetry data commonly feature high spatial and temporal autocorrelation. This can be present in locations, aspects of behavior or properties of the movement trajectory, and environmental variables collected in association with locations. The dive record of each individual was checked for spatial and temporal autocorrelation in maximum depth of consecutive dives using the autocorrelation function (ACF) (acf function, base package, R Development Core Team 2010). Gray seals do not dive at a constant rate (number of dives per unit time), so to view the autocorrelation in a more standardized temporal context, ACF plots of the raw data were constructed for the daily mean maximum dive depth of each individual (Fig. 1). The pattern of autocorrelation was not modeled; instead, it was dealt with by subsampling the data set. Taking every tenth dive from the track of each individual reduced the autocorrelation substantially. The resulting data set consisted of 21,986 dives from all trips from seven individuals (Fig. 2).

\section{Statistical Modeling}

A GAMM was fitted to the maximum dive depth time-series to generate spatial predictions of maximum dive depth. Individual was included as a random effect, to
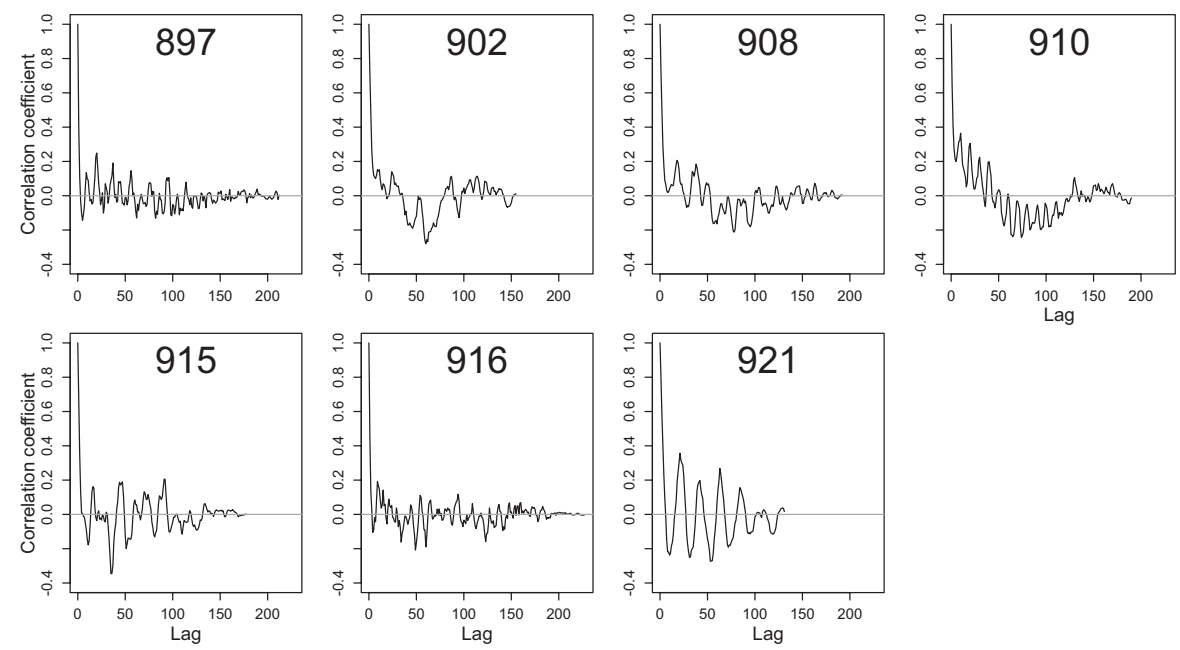

Figure 1. The autocorrelation function (ACF) of the daily mean in maximum dive depth for consecutive dives of each individual. The lag is measured in days from tagging. 

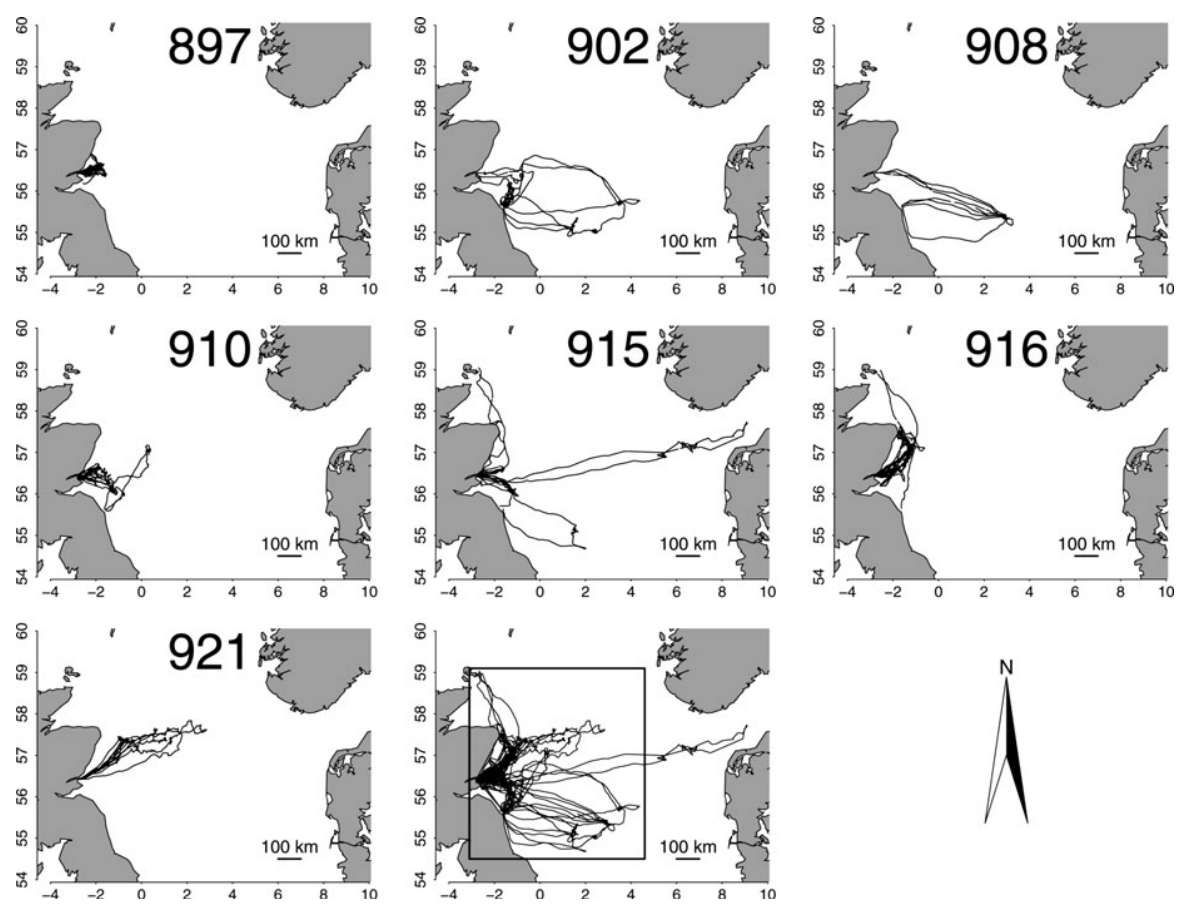

Figure 2. Maps of the dive locations during trips to sea of seven gray seals instrumented in 2008. Map coordinates are in latitude and longitude (WGS84 projection). The seventh plot shows all tracks overlaid, and a black box indicating the extent of the area used to make predictions.

capture the individual-level variability in dive depth. The explanatory variables included in the model were selected from the available variables on the premise that they characterize some aspects of the spatial, physical, and temporal environment that seals experience while at sea (bathymetry, horizontal displacement, latitude and longitude, Julian day, sediment composition, light conditions), and individual characteristics of the tagged animals (individual identity). Sex and morphometrics were not used as explanatory variables. Knowledge of the diet of this population is incomplete and distribution data on known prey species is sparse. Instead, the percentage of gravel in the sediment was used as a proxy for the potential presence of sand eels, which are known to form a substantial part of seal diet on the east coast of the UK and western North Atlantic from scat sample analysis (Prime and Hammond 1990; Bowen and Harrison 1994; Hammond et al. 1994a, b). The variables used to explain maximum dive depth in the final model were bathymetry (range $-4.0-200.9 \mathrm{~m}$, source: DigBath250 data set, scale 1:250 000, British Geological Society), longitude in WGS84 decimal degrees (range 3.04-9.10), latitude in WGS84 decimal degrees (range 54.7-59.0), rate of horizontal displacement between surface locations (range $0.00-2.20 \mathrm{~m} / \mathrm{s}$ ), Julian day (range 100-334), percentage gravel in the sediment (range 0.00-83.0), and a binary variable for light conditions (daylight/darkness). A variable for "individual" was included as a random effect, with the assumption that the sample of individual seals, albeit small, is representative of the variability in maximum dive depth characteristics of this population, and that their behavior is not more similar than would be expected by chance. Although the dives in this data set 
come from just seven individuals, there was no reason to expect their diving behavior to be correlated.

Longitude, latitude, horizontal displacement rate, Julian day, and light conditions were taken directly from the data delivered by the tag or derived from them. Light conditions at the time of the dive were calculated as a binary variable (light or dark), based on the timing of each dive relative to local sunrise and sunset, using the sunriset function in the maptools library (Lewin-Koh and Bivand 2011) in R.

The bathymetry and sediment data were processed as per Aarts et al. (2008), resulting in values on a $1 \mathrm{~km}$ grid for both variables. Bathymetry and sediment type were matched to dive locations using bilinear weights to interpolate values on a rectangular grid to irregular locations, so that interpolated values at dive locations were interpolated along the $\mathrm{x}$ and $\mathrm{y}$ axes (latitude and longitude) from the four nearest points in the grid of bathymetry and sediment values. This was carried out using the interp.surface function in the fields package (Furrer et al. 2011) in R.

The nonlinear relationships between the response and many of the explanatory variables, and the potential for systematic variation in model residuals for dives made by the same animal, were accounted for by fitting a GAMM. Although the structure of the response data was such that there were two levels of nesting, dives from trips within individuals, a single random effect was included for individual, because the depth characteristics of dives from different trips within and between individuals were found to vary little.

The GAMM was implemented with the gam function in the mgcr library (Wood 2006, 2008) in R using fast restricted maximum likelihood (fREML) as the fitting method. The random effect for individual was implemented using the "re" smoother option, which is appropriate for simple, independent random effects (Wood 2011). Under this formulation random effects are implemented by applying a penalty to the model matrix in the form of a scalar multiple of the identity matrix, hence assuming that the coefficients associated with the penalty are independent and normally distributed (Wood 2008). The "gamma" parameter of the gam function (effectively a roughness penalty) was set to 1.4 (Wood 2006), to reduce the chance of over-fitting to the data. Latitude and longitude entered the model as a spherical smooth function of the response using the "sos" two-dimensional isotropic smoother option in the mgcv library, with a first derivative penalty, which is the default, and 100 knots. All other continuous variables were fitted as smoothed functions with a minimum of $6(k=6)$ and a maximum of 10 knots $(k$ $=10)$ and daylight/darkness entered the model as a factor. The appropriate number of knots in each case was chosen using the routines outlined in the mgcr package manual and Wood (2006). Cubic regression splines ("cs"; this type of smooth incorporates a smooth modification technique called shrinkage, which is discussed below) were used as the basis functions for all continuous variables.

Maximum dive depth recorded by the tag is a strictly positive, real-valued response variable, so a gamma error distribution, which ensures positive predictions and assumes that errors will be small near zero, was initially considered to be the most appropriate. However, both the bathymetric measurements and dive locations are subject to some degree of spatial measurement error. This can result in a mismatch between dive depth and apparently available bathymetry and is most obvious for dives within coastal grid cells where water depth is shallow. Because of this, errors will not be smallest near zero depth, and the normal distribution was considered a reasonable alternative. Two important practical advantages of using a Normal error structure are shorter fitting time and availability of exact, rather than approximate 
estimation of confidence intervals (Wood 2006). A model with a Normal error distribution and an identity link function was used.

\section{Variable Selection}

A generalized additive model describes the response data as a smooth, nonlinear function of the covariates. To prevent over-fitting, any excessive "wiggliness" in the fitted model must be penalized. A strict penalization of the response to any given covariate can shrink (i.e., lessen) its effect without removing it from the model. Shrinkage smoothing methods allow smooth components to be shrunk to zero during smoothness selection, effectively extinguishing their effect from the model (Wood 2008). The appeal of shrinkage approaches is that (1) they have the consistency of methods that explore the combination of all possible subsets of covariates (subset selection); (2) with them, variable selection can be achieved in a single step; and (3) they explore a larger part of model space than stepwise model selection procedures because they do not remove (or include) a variable permanently and hence they always give the opportunity to all the variables to increase (as well as decrease) their contribution to the model. As a result, shrinkage approaches do not suffer from the uncertainty inherent in stepwise variable selection procedures and model subset selection (Marra and Wood 2011).

The mgcr library offers two methods for the modification of smooths that employ a shrinkage approach: "double penalty" and "shrinkage." The double penalty approach was used because of its stability in prediction, and the advantage of being able to use it with any spline basis, including spherical splines. Despite it requiring twice the number of smoothing parameters to be estimated (Marra and Wood 2011), model fitting with the double penalty approach was still quick (approximately 2 min) on a machine with two $2.8 \mathrm{GHz}$ quad-core Intel Xeon processors and $8 \mathrm{~GB}$ of RAM.

If penalization is strong enough, shrinkage smoothers will push all the coefficients of the smooth to zero, cancelling out its effect (Wood 2008). Hence, with increasing shrinkage, a model that contains the (shrunk) covariate tends to become equivalent to a model that does not.

\section{Predictions}

To create individual prediction maps that could be meaningfully combined, given the different spatial coverage and longitudinal extent of their tracks and dive records, the prediction grid for each individual was set up to have a resolution of approximately $1 \mathrm{~km}^{2}$. The spatial extent of predictions with respect to data points was limited to within $3 \%$ of the latitudinal and longitudinal range of the data (gam option too.far $=0.03$ ). Prediction matrices were constructed for bathymetry at the resolution of the prediction grid. Four composite prediction maps were generated by combining the seven individual prediction maps in each case: two maps of predicted maximum dive depth, during light and dark conditions, and two maps of predicted maximum depth relative to available depth (i.e., proportional use of the water column), during light and dark conditions. Predicted proportional use of the water column was calculated as the ratio of predicted maximum dive depth to bottom depth at the dive location. Where the predicted maximum dive depth was greater than the bottom depth measurement, the ratio was set to one. 
Julian day was set to 160 (8 June 2008), horizontal displacement to $0.3 \mathrm{~m} / \mathrm{s}$, which was the median value in the data. Predictions were made for each individual animal over a region containing the majority of track data (Fig. 2; longitudinal range $3.1^{\circ}-$ $4.0^{\circ}$, latitudinal range $54.5^{\circ}-59.1^{\circ}$ ), scaling each prediction matrix by the proportional contribution of each individual to the data set, in terms of number of dives. The seven scaled prediction matrices were then summed to produce population-level prediction maps, as described above.

\section{RESUlTS}

There was a persistent cyclic autocorrelation pattern in the raw data for all individuals, with successive increases and decreases in similarity between dive depths with increasing time lag. Some individuals reliably returned to the same areas and by similar routes during different trips (e.g., 897, Fig. 2) while others interspersed trips to regularly used areas, with long trips to distant or different areas (e.g., 915, Fig. 2). On the whole, all individuals used a small number of geographic regions frequently, with some overlap between individuals. There was little evidence for similarity or synchronicity in the pattern of autocorrelation present in the time series of depths from different individuals based on spectral density analysis of the dominant frequency of autocorrelation. This pattern was less pronounced in the thinned raw data, and there was no serial pattern in the model residuals.

The plots of the component smooth functions (Fig. 3) show the overall relationship between maximum dive depth and each of the covariates, under the model. Maximum dive depth had a clear, positive relationship with bathymetry until approximately $60 \mathrm{~m}$. The negative relationship apparent at the upper end of the bathymetric range is largely unsupported by the data, which are concentrated in the range $0-100 \mathrm{~m}$. The smooth for horizontal displacement (speed) suggests that animals dive less deeply as horizontal displacement increases. The relationship with Julian day was variable, with the greatest maximum dive depth being reached in July (ca. day 200).

There was a positive relationship between maximum dive depth and the percentage of gravel in the sediment up to $20 \%$ and a negative relationship with large standard errors, with increasing gravel content, thereafter. The plot for the random effect for individual shows that although five out of seven animals had similar maximum dive depth characteristics, under the model, two of these (902 and 915, a male and female) had more extreme effects than the rest. Overall, the size of the individual effect was relatively small compared to the fixed effects, judging by the range of the $y$-axis. The partial residual plot for light conditions shows that the predicted maximum depth for dives made in daylight was deeper than for those made during the hours of darkness.

The model captured $37 \%$ of the variability (model deviance) in the data. The distribution of the residuals was, on the whole, symmetrical and centered on zero and there was no evidence for autocorrelation. There was some evidence of over-prediction in a small peak of negative residuals, which was also obvious in plots of observed $v$. fitted values of maximum dive depth.

The data suggest that the seals dived to the deepest available depth some of the time but that they also performed shallow dives over deep water throughout the bathymetric range they encountered. An area used by three of the seven individuals 

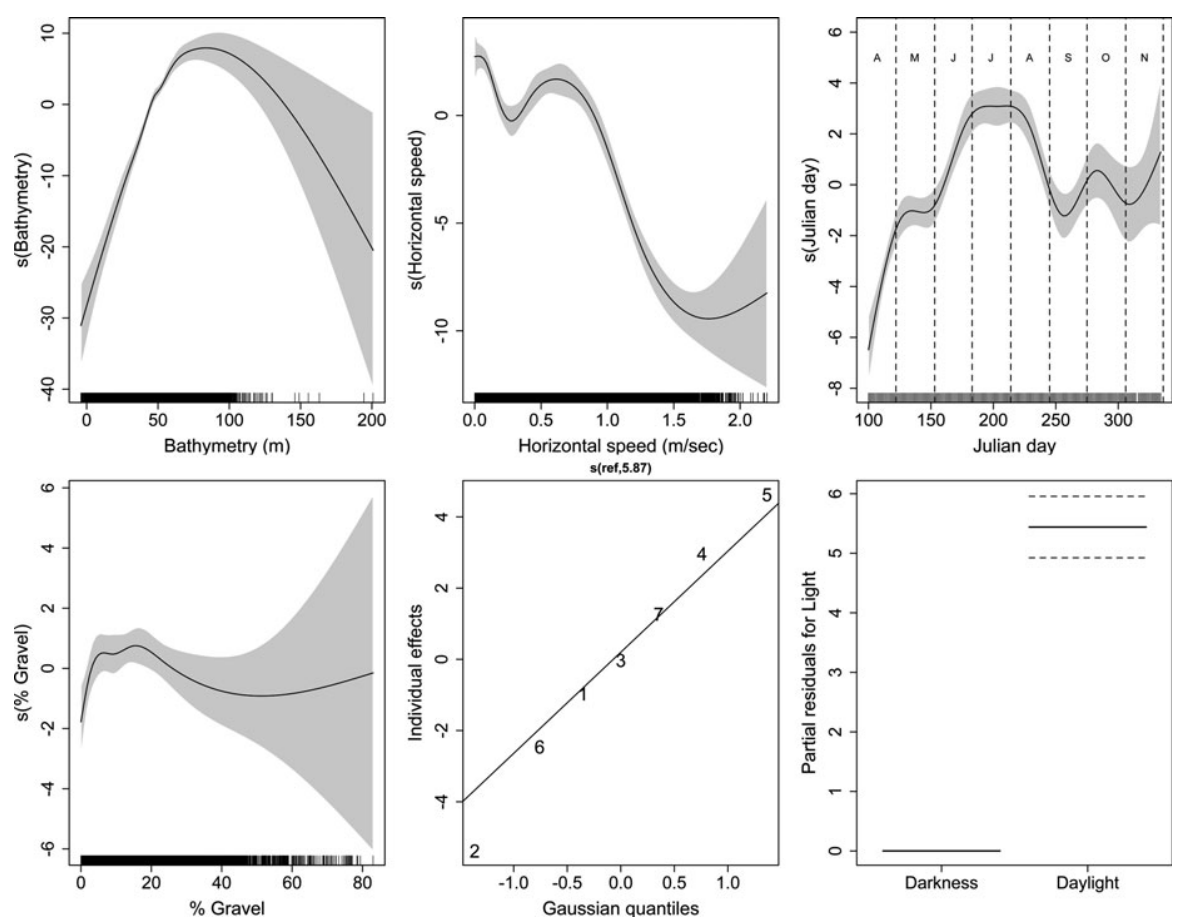

Figure 3. The relationship between maximum dive depth and each explanatory variable in the model except latitude and longitude, including smooth terms, the random effect for individual and factor variable for light conditions. The gray shading in plots of smooth functions extends two standard errors either side of the smooth. The dashed vertical lines and letters in the plot for Julian day show months April (A) to November $(\mathrm{N})$. The numbers in the plot for Individual effects relate to the individual id number (where 1 to 7 correspond to 897 to 921 , see Table 1).

during the deployment period (two males, 902 and 908, and the largest of the four females, 915, Fig. 2) was Dogger Bank, to the southeast of Abertay Sands, at approximately $55^{\circ} \mathrm{N}, 2^{\circ} \mathrm{W}$, where the model predicted that all three seals dived to the seabed. Two females (897 and 910, Fig. 2) used an area approximately $100 \mathrm{~km}$ west/northwest of Abertay Sands, and were also predicted to dive to the seabed (Fig. 4).

The spatial predictions of maximum dive depth show a vertical band spanning the latitudinal range of the tracks, in which all seals appear to dive deeper both in light and dark conditions (Fig. 4A, B). Overall, dives made during darkness were predicted to be shallower across the whole area (Fig. 4B). The pattern of proportional use of the maximum available depth showed consistent predictions of diving to $70 \%-100 \%(0.7-1.0)$ of the available depth during daylight (Fig. 4C). When diving during darkness, animals utilized proportionally less of the available depth and dives were also shallower in absolute terms (Fig. 4D). This pattern varied spatially. Maximum dive depth was predicted to exceed $80 \%$ of the available depth only $25 \%$ of the time during darkness, compared to $46 \%$ during daylight. It would seem that these predictions indicate a shift to mid-water dives $(60 \%-80 \%$ of available depth) during darkness ( $57 \%$ vs. $44 \%$ during daylight). Seals were also 


\section{Spatial variation in maximum dive depth}
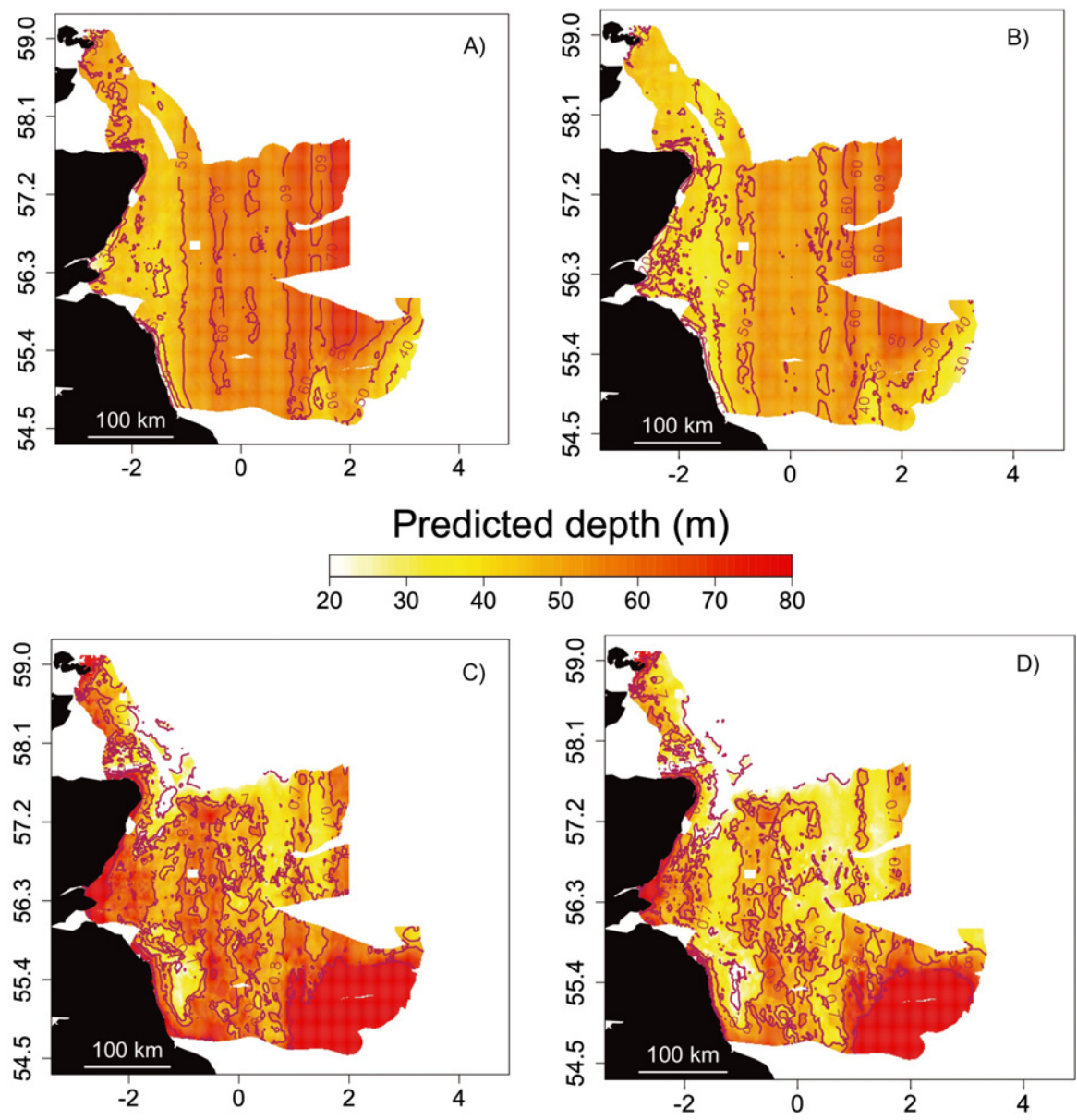

Predicted depth/Available depth

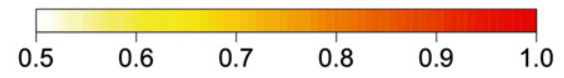

Figure 4. Map (latitude vs. longitude) of model predictions for all individuals combined on a $1 \mathrm{~km}^{2}$ grid. Map A shows maximum dive depth predictions during daylight, and B during darkness. Map C shows predicted relative maximum dive depth (maximum dive depth divided by available depth) during daylight, and D during darkness.

predicted to spend more time in the upper part of the water column (less than $60 \%$ of available depth) during darkness ( $18 \%$ vs. $10 \%$ during daylight). There were some areas where there was no change in absolute maximum dive depth or proportional dive depth, the most distinct of which was the Dogger Bank. 


\section{Discussion}

Spatial predictions of marine mammal distribution and habitat preference are most commonly presented in two dimensions, latitude and longitude. For diving animals, this is an inadequate representation of their use of space. We constructed a regression model to explain the conditions under which gray seals in the western North Sea dive to different maximum depths, based on a thinned data set of 21,986 dives from seven individuals that were instrumented with GPS phone tags on the east coast of the UK in April 2008. The model explained 37\% of the variability in the response data overall, and predictions were poor at the extremes of the observed range of the response variable, particularly when shallow dives were performed over deep water.

Descriptive metrics of diving, foraging behavior, and movement characteristics are often used in place of raw data (e.g., Schreer and Testa 1996, Fauchald and Tveraa 2003). These can be hard to interpret biologically without independent verification of function or their suitability as proxies for what they are intended to convey. The motivation for using maximum dive depth as the response variable was to investigate the usefulness of this very simple aspect of depth use in a benthic diver, for describing the relationship between diving behavior, environmental, spatiotemporal and individual covariates.

Given the strong association between sand eels, a primary prey type for benthic foragers such as gray seals and diving seabirds, and coarse sand and fine to medium gravel (Wright et al. 1998, van der Kooij et al. 2008), sediment type could be considered as a proxy for the availability of prey resources to species like gray seals, that feed on benthic or demersal prey (Wanless et al. 1993, Gremillet et al. 1998, McConnell et al. 1999, Aarts et al. 2008, Watanuki et al. 2008). However, sand eels are not benthic divers' only prey. The gray seals in this data set used primarily three water masses, as defined by Ehrlich et al. (2009): Scottish Coastal Water, North Atlantic Water, and Northern North Sea Water. Prey types represented in scat samples from the east coast of Scotland are probably only representative of prey taken by seals in Scottish Coastal Water. Trawls carried out in North Atlantic and Northern North Sea Water found that two gadids, haddock (Melanogrammus aeglefinus) and whiting (Merlangius merlangus) dominated their catches (Ehrlich et al. 2009). Although this cannot be used as evidence that gray seals consume these species, haddock and whiting also prey on sand eels and other species consumed by gray seals. Whiting in particular has been found to make up a large proportion of diet by weight late in the year based on scat sample analysis from Orkney (Hammond et al. 1994a).

Data with inherent spatial and temporal structure, such as consecutive dive depths, are most often correlated in space and time. Since seals often dive to the bottom, and neighboring depths are more similar, we expected a spatiotemporal pattern in the spatial time-series of maximum dive depth. However, bathymetry and the spatial coordinates of each dive were included in the model covariates, and we expected them to capture most of this variability. A possible source of periodicity in dive depth, especially given the different pattern present in each individual, is an inherent rhythm in the behavior of each animal, e.g., due to digestion times and individual diet, which was not captured by the explanatory variables used here. The strong periodic pattern that was observed in the raw data, and model residuals from the full data set, before thinning, and its failure to decay with increasing time lag, could also be due to onshore-offshore movement, as a result of multiple trips during the tracking period. 
In this analysis, autocorrelation was dealt with by subsampling, resulting in loss of information about the time-series of dive depths immediately prior to a dive. It has been shown that gray seal diving behavior occurs in bouts and that there are different types of dive bouts (Beck et al. 2003c, Austin et al. 2006). The temporal scale considered in this analysis was that of individual dives and trips, but not the structure of dives within trips. It is likely that explicitly modeling the bout structure of seal diving behavior would lead to improved predictive power. Another temporal effect that might have contributed to dive depths being over-predicted at shallow depths might be the trip classification routine adopted here. The $3 \mathrm{~h}$ haul-out definition used to separate trips is likely to have excluded short trips in the vicinity of the haul-out, during which opportunistic foraging should not be ruled out. The individual whose dives were also most accurately predicted by the model, was the one that carried out the most trips in this study and whose dive depth at shallow depths was best represented (897, see Fig. 2).

External features of the physical environment both in space and time (light/dark conditions, bathymetry, season, geographic coordinates) are considered to be adequately represented in the model, although tidal effects and currents, water temperature, and stratification were not included and could have also explained some of the variability. Individual, internal factors that were not considered here and have been shown to affect diving behavior in gray seals include hormonal and metabolic status of the individuals, body mass, body condition, and the associated locomotory implications (buoyancy) (Beck et al. 2000, 2003a, b, c).

These poorly predicted shallow dives made over deep water could be resting or digesting dives, since it has been documented for gray seals in captivity that food processing can be delayed for many hours after a feeding event (Sparling et al. 2007).

The negative effect of darkness on dive depth found here could be the result of the model inaccurately predicting a unimodal mean for bimodal night time diving behavior. If deep feeding dives are interspersed by shallow processing or resting dives, the effect could be an intermediate predicted dive depth. Alternatively, if seals feed at or just above the seabed during the day, the positive effect of daylight on dive depth ties in with the diurnal behavior of sand eels, which feed in the water column in the day and bury themselves in the sediment at night (van der Kooij et al. 2008), though there is anecdotal evidence that they can also spend time buried during daylight hours. It is possible that when targeting sand eels or other vertically migrating prey, gray seals dive deeper in the day and spend the hours of darkness carrying out shallower dives during which other metabolic functions can be carried out, or even feeding on other prey.

The effect of light conditions on maximum dive depth and proportional use of the water column disappeared for dives over the Dogger Bank, a fishing ground for lesser sand eels (Ammodytes marinus) (van der Kooij et al. 2008). Seals consistently dive to the seabed in this area, according to model predictions. If shallow night time dives relate to the type of prey seals are exploiting, this lack of diurnal change in dive depth on the Dogger Bank might suggest that they are feeding on other prey that are available when sandeels are not. Alternatively, if shallow night time dives are processing dives, it might suggest that this area is shallow enough to enable seals to carry out metabolic functions while diving to the seabed. There is anecdotal evidence that seals continue to exploit sandeels at night when sand eels are buried in the sediment and more predictably concentrated by disturbing the sediment. This might also explain continued dives to the seabed in this relatively shallow area. The relationship between dive duration and light conditions was not 
investigated here, but given the diurnal effect on dive depth, dive duration may follow a similar pattern.

To summarize, the effect of environmental, behavioral, and individual characteristics on maximum dive depth was investigated using a data set of trips from seven animals. A small number of covariates explained a non-negligible proportion of the variability in the data, even though no intrinsic variables were considered. Although, by design, this analysis does not examine the causal relationship behind dive depth, i.e., the mechanism that drives the alternation between shallow and deep dives, we show that this aspect of vertical space use in the marine environment can be described and predicted with commonly available data on spatiotemporal covariates. This is useful as a baseline for understanding depth use using regression models, and could be used as the first step in a two-part regression model for depth use, for explaining distribution of depths, given the maximum depth visited. Depth aside, longer dives might suggest more profitable, higher quality patches in the wild as has been found in captivity (Sparling et al. 2007), which could be investigated further.

The variability in the effect of the spatial and temporal covariates (bathymetry, Julian day, light conditions, gravel) suggests that foraging effort is spatially and temporally heterogeneous between individuals. However, the scale of the vertical axis of the smooth functions for the fixed effects, particularly bathymetry, horizontal speed, and Julian day, relative to the random effect suggests that the effect of individual variability captured by this covariate is relatively small compared to environmental effects.

With this analysis of gray seal diving we illustrate the use of a mainstream statistical method, a GAMM, to generate three-dimensional maps of dive depth in marine animals. These maps of spatial predictions of maximum dive depth, an aspect of depth use, contribute to knowledge of diving species' biology, and make it possible to estimate and visualize the potential rate of interaction between diving animals and subsurface developments, such as marine renewable energy installations (Furness et al. 2012). This approach is relevant to plunge and pursuit diving seabirds (e.g., gannets, Sula spp., and cormorants, Phalacrocorax spp.), pinnipeds, and cetaceans that focus the effort at the deepest part of their dives. Usage maps based on spatial predictions of the distribution of maximum dive depth can be generated in this way for large areas, provided dive data and environmental data are available. An integration of two-dimensional maps of habitat use and maps of dive depth would provide a more complete view of diving animals' space use and movement ecology.

\section{ACKNOWLEDGMENTS}

The telemetry data presented in this manuscript were collected as part of a project funded by the UK Department for Business, Enterprise and Regulatory Reform (now Department of Energy and Climate Change). Fieldwork was carried out under an Animals (Scientific Procedures) Act 1986 Project License PPL 60/3303. The authors are grateful to the principal investigator of the project, Bernie McConnell, SMRU, for making the data available, and to those involved in the fieldwork. Theoni Photopoulou was funded by SMRU Ltd in the form of a Ph.D. studentship, 2008-2012. The authors wish to thank Sophie Smout and Carol Sparling for providing useful comments on earlier versions of the manuscript, as well as two anonymous reviewers for their constructive comments. 


\section{Literature Cited}

Aarts, G., M. Mackenzie, B. McConnell, M. Fedak and J. Matthiopoulos. 2008. Estimating space-use and habitat preference from wildlife telemetry data. Ecography 31:140-160.

Austin, D., W. D. Bowen, J. I. McMillan and S. J. Iverson. 2006. Linking movement, diving, and habitat to foraging success in a large marine predator. Ecology 87:30953108.

Beck, C. A., W. D. Bowen and S. J. Iverson. 2000. Seasonal changes in buoyancy and diving behavior of adult gray seals. Journal of Experimental Biology 203:2323-2330.

Beck, C. A., W. D. Bowen, J. I. McMillan and S. J. Iverson. 2003a. Sex differences in the diving behavior of a size dimorphic capital breeder: The gray seal. Animal Behavior 66:777-789.

Beck, C. A., W. D. Bowen and S. J. Iverson. 2003b. Sex differences in the seasonal patterns of energy storage and expenditure in a phocid seal. Journal of Animal Ecology 72:280-291.

Beck, C. A., W. D. Bowen, J. I. McMillan and S. J. Iverson. 2003c. Sex differences in diving at multiple temporal scales in a size-dimorphic capital breeder. Journal of Animal Ecology 72:979-993.

Beck, C. A., S. J. Iverson, W. D. Bowen and W. Blanchard. 2007. Sex differences in gray seal diet reflect seasonal variation in foraging behavior and reproductive expenditure: Evidence from quantitative fatty acid signature analysis. Journal of Animal Ecology 76:490-502.

Bowen, D. W., and G. D. Harrison. 1994. Offshore diet of gray seals Halichoerus grypus near Sable Island, Canada. Marine Ecology Progress Series 112:1-11.

Bowen, D. W., J. W. Lawson and B. Beck. 1993. Seasonal and geographic variation in the species composition and size of prey consumed by grey seals (Halichoerus grypus) on the Scotian Shelf. Canadian Journal of Fisheries and Aquatic Sciences 50:1768-1778.

British Geological Society. DigBath250. [Data set]. Available at http://www.bgs.ac.uk/ products/digBath250/home.html.

Ehrlich, S., V. Stelzenmüller and S. Alderstein. 2009. Linking spatial pattern of bottom fish assemblages with water masses in the North Sea. Fisheries Oceanography 18:36-50.

Fauchald, P., and T. Tveraa. 2003. Using first-passage time in the analysis of area-restricted search and habitat selection. Ecology 84:282-288.

Fedak, M. A., S. S. Anderson and M. G. Curry. 1983. Attachment of a radio tag to the fur of seals. Journal of Zoology 200:298-300.

Fedak, M. A., P. Lovell and B. J. McConnell. 1996. MAMVIS: A marine mammal behavior visualization system. Journal of Visualization and Computer Animation 7:141-147.

Furness, R. W., H. M. Wade, A. M. C. Robbins and E. A. Masden. 2012. Assessing the sensitivity of seabird populations to adverse effects from tidal stream turbines and wave energy devices. ICES Journal of Marine Science 69:1466-1479.

Furrer, R., D. Nychka and S. Sain. 2011. fields: Tools for spatial data. R package version 6.6. Available at http://CRAN.R-project.org/package=fields.

Google, Inc. 2009. Google Earth (Version 3.0.11733). Available at http://www.google.com/ earth/index.html.

Gremillet, D., G. Argentin, B. Schulte and B. M. Culik. 1998. Flexible foraging techniques in breeding cormorants Phalacrocorax carbo and Shags Phalacrocorax aristotelis: Benthic or pelagic feeding? Ibis 140:113-119.

Hammond, P. S., A. J. Hall and J. H. Prime. 1994a. The diet of gray seals around Orkney and other island and mainland sites in north-eastern Scotland. Journal of Applied Ecology 31:340-350.

Hammond, P. S., A. J. Hall and J. H. Prime. 1994b. The diet of gray seals in the Inner and Outer Hebrides. Journal of Applied Ecology 31:737-746.

Kanatous, S. B., L. V. DiMichelle, D. F. Cowan and R. W. Davis. 1999. High aerobic capacities in the skeletal muscles of pinnipeds: adaptations to diving hypoxia. Journal of Applied Physiology 86:1247-1256. 
Kooyman, G. L., and P. J. Ponganis. 1998. The physiological basis of diving to depth: Birds and mammals. Annual Review of Physiology 60:19-32.

Marra, G., and S. N. Wood. 2011. Practical variable selection for generalized additive models. Computational Statistics and Data Analysis 55:2372-2387.

McConnell, B. J., M. A. Fedak, P. Lovell and P. S. Hammond. 1999. Movements and foraging areas of gray seals in the North Sea. Journal of Applied Ecology 36:573-590.

Meir, J. U., C. D. Champagne, D. P. Costa, C. L. Williams and P. J. Ponganis. 2009. Extreme hypoxemic tolerance and blood oxygen depletion in diving elephant seals. American Journal of Physiology Regulatory, Integrative and Comparative Physiology 297:927939.

Lewin-Koh, N. J., R. Bivand, contributions by E. J. Pebesma, E. Archer, A. Baddeley, et al. 2011. maptools: Tools for reading and handling spatial objects. $\mathrm{R}$ package version $0.8-$ 10. Available at http://CRAN.R-project.org/package=maptools.

Prime, J. H., and P. S. Hammond. 1990. The diet of gray seals from the south-western North Sea assessed from analyses of hard parts found in faeces. The Journal of Applied Ecology 27:435-447.

R Development Core Team. 2010. R: A language and environment for statistical computing. R Foundation for Statistical Computing, Vienna, Austria.

Schreer, J. F., and J. W. Testa. 1996. Classification of Weddell seal diving behavior. Marine Mammal Science 12:227-250.

Sparling, C. E., and M. A. Fedak. 2004. Metabolic rates of captive gray seals during voluntary diving. The Journal of Experimental Biology 207:1615-1624.

Sparling, C. E., M. A. Fedak and D. Thompson. 2007. Eat now, pay later? Evidence of deferred food-processing costs in diving seals. Biology Letters 3:94-98.

Thompson, D., P. S. Hammond, K. S. Nicholas and M. A. Fedak. 1991. Movements, diving and foraging behavior of gray seals (Halichoerus grypus). Journal of Zoology 224:223-232.

van der Kooij, J., B. E. Scott and S. Mackinson. 2008. The effects of environmental factors on daytime sandeel distribution and abundance on the Dogger Bank. Journal of Sea Research 60:201-209.

Wanless, S., T. Corfield, M. P. Harris, S. T. Buckland and J. A. Morris. 1993. Diving behavior of the shag Phalacrocorax aristotelis (Aves: Pelecaniformes) in relation to water depth and prey size. Journal of Zoology 231:11-25.

Watanuki, Y., F. Daunt, A. Takahashi, M. Newell, S. Wanless, K. Sato and N. Miyazaki. 2008. Microhabitat use and prey capture of a bottom-feeding top predator, the European shag, shown by camera loggers. Marine Ecology Progress Series 356:283-293.

Wood, S. N. 2006. Generalized additive models: An introduction with R. CRC Press, Boca Raton, FL.

Wood, S. N. 2008. Fast stable direct fitting and smoothness selection for generalized additive models. Journal of the Royal Statistical Society B 70:495-518.

Wood, S. N. 2011. Fast stable restricted maximum likelihood and marginal likelihood estimation of semiparametric generalized linear models. Journal of the Royal Statistical Society B 73:3-36.

Wright, P. J., S. S. Pedersen, C. Anderson, P. Lewy and R. Proctor. 1998. The influence of physical factors on the distribution of lesser sand eels, Ammodytes marinus, and its relevance to fishing pressure in the North Sea. ICES Document CM 1998/AA: 03. 18 pp.

Received: 8 March 2013

Accepted: 30 September 2013 